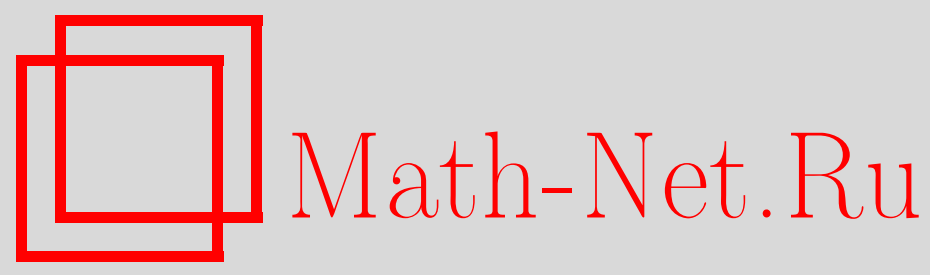

В. А. Мильман, Продолжение функций, сохраняющее модуль непрерывности, Матем. заметки, 1997, том 61, выпуск 2, 236-245

DOI: https://doi.org/10.4213/mzm1496

Использование Общероссийского математического портала Math-Net.Ru подразумевает, что вы прочитали и согласны с пользовательским соглашением http://www . mathnet.ru/rus/agreement

Параметры загрузки:

IP : 54.198 .67 .100

26 апреля 2023 г., 15:38:29

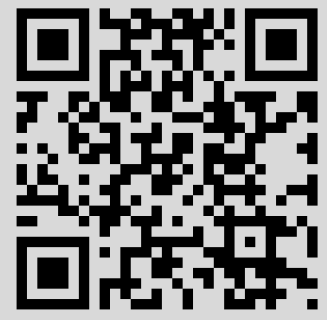




\section{ПРОДОЛЖЕНИЕ ФУНКЦИЙ, СОХРАНЯЮЩЕЕ МОДУЛЬ НЕПРЕРЫВНОСТИ}

\section{В. А. Мильман}

Рассмотрена задача продолжения действительнозначной функции с подмножества метрического пространства на все пространство. Предложен оператор продолжения, обеспечивающий сохранение модуля непрерывности функции, и исследованы его свойства. Приведено приложение к задаче о следе локально липшицевой функции на компактном подмножестве метрического пространства.

Библиограффия: 6 названий.

Рассматривается задача продолжения действительнозначной функции с подмножества метрического пространства на все пространство с сохранением модуля непрерьвности функции. Подобные задачи возникают в ряде вопросов математического анализа и геометрии [1, с. 220], [2], [3].

1. Используемые обозначения и понятия. Будем придерживаться следующих обозначений:

$\mathbb{R}_{+}-$множество неотрицательных действительных чисел,

$(X, d)$ - метрическое пространство,

$D$ - ограниченное подмножество множества $X$,

$\bar{D}$ - замыкание подмножества $D$,

$f: D \rightarrow \mathbb{R}$ - ограниченная функция,

$\omega: \mathbb{R}_{+} \rightarrow \mathbb{R}_{+}-$неубьвающая функция.

Функция $\omega$ назьвается полуаддитивной, если

$$
\omega(a+b) \leqslant \omega(a)+\omega(b) \quad \forall a, b \geqslant 0 .
$$

Функция $\omega$ назьвается модулем непрерывности [4], если она неубывающая, полуаддитивная и

$$
\omega(0)=\lim _{t \rightarrow 0} \omega(t)=0 .
$$

Определим функциональные классы

$$
\begin{gathered}
m * \operatorname{Lip}(D)=\{f: D \rightarrow \mathbb{R}|\forall x, y \in D| f(x)-f(y) \mid \leqslant m \cdot d(x, y)\} \\
H(D, \omega)=\{f: D \rightarrow \mathbb{R}|\forall x, y \in D| f(x)-f(y) \mid \leqslant \omega(d(x, y))\} .
\end{gathered}
$$

Первьй из классов состоит из функций с константой Липшица, не превосходящей $m$, второй - из функций, модуль непрерывности которых имеет заданную мажоранту $\omega$. $\Phi$ ункция $\omega$ не обязана быть модулем непрерьвности. 
Относительной константой Лишшица функции $h: X \rightarrow \mathbb{R}$ в точке $x$ будем назьвать величину

$$
k(h, x)=\sup \left\{\frac{|h(x)-h(z)|}{d(x, z)} \mid z \in X, z \neq x\right\} .
$$

2. Постановка задачи. Пусть заданы неубывающая функция $\omega$ и ограниченная функция $f \in H(D, \omega)$. Требуется продолжить $f$ на множество $X$, т.е. найти такую функцию $g: D \rightarrow \mathbb{R}$, чтобы $g(x)=f(x) \forall x \in D$ и $g \in H(X, \omega)$.

Если функция $\omega$ является модулем непрерьвности, известны [3], [5] операторы продолжения $E_{1}, E_{2}: H(D, \omega) \rightarrow H(X, \omega)$, то

$$
\begin{aligned}
& E_{1}(f)(x)=\sup \{f(y)-\omega(d(y, x)) \mid y \in D\} \\
& E_{2}(f)(x)=\inf \{f(y)+\omega(d(y, x)) \mid y \in D\}
\end{aligned}
$$

В настоящей работе строится иной оператор продолжения и исследуются его свойства.

3. Вспомогательные функции. Определим функции $u_{f}, v_{f}:(X \backslash \bar{D}) \times \mathbb{R} \rightarrow \mathbb{R}$ :

$$
u_{f}(x, c)=\sup \left\{\frac{c-f(y)}{d(x, y)} \mid y \in D\right\}, \quad v_{f}(x, c)=\sup \left\{\frac{f(y)-c}{d(x, y)} \mid y \in D\right\}, \quad x \in X \backslash \bar{D} .
$$

Лемма 1. Если $x \in X \backslash \bar{D}$, то уравнение

$$
u_{f}(x, c)=v_{f}(x, c)
$$

имеет единственное решение $c_{0}=c_{0}(x)$. При этом для $c \neq c_{0}$

$$
\sup \left\{\frac{\left|c_{0}-f(y)\right|}{d(x, y)} \mid y \in D\right\}<\sup \left\{\frac{|c-f(y)|}{d(x, y)} \mid y \in D\right\} \text {. }
$$

ДокАЗАТЕЛЬСтво. Из ограниченности множества $D$ и того, что $x \notin \bar{D}$ следует, что

$$
b_{1}=\sup \{d(x, y) \mid y \in D\}<+\infty, \quad b_{2}=\inf \{d(x, y) \mid y \in D\}>0 \text {. }
$$

Из определения функции $u_{f}$ легко получаются неравенства

$$
\begin{aligned}
& u_{f}(x, c)+\frac{a}{b_{1}} \leqslant u_{f}(x, c+a) \leqslant u_{f}(x, c)+\frac{a}{b_{2}}, \quad \text { где } a>0 . \\
& u_{f}(x, c)-\frac{a}{b_{2}} \leqslant u_{f}(x, c-a) \leqslant u_{f}(x, c)-\frac{a}{b_{1}},
\end{aligned}
$$

Из них следует непрерывность и строгое возрастание функции $u_{f}$ по $c$, а также равенства

$$
\lim _{c \rightarrow+\infty} u_{f}(x, c)=+\infty, \quad \lim _{c \rightarrow-\infty} u_{f}(x, c)=-\infty .
$$

Аналогично доказьвается непрерьвность и убывание функции $v_{f}$ по $c$, а также аналогичные равенства. Следовательно, функция $u_{f}(x, c)-v_{f}(x, c)$ непрерывна и строго возрастает по $с$ и

$$
\lim _{c \rightarrow+\infty}\left(u_{f}(x, c)-v_{f}(x, c)\right)=+\infty, \quad \lim _{c \rightarrow-\infty}\left(u_{f}(x, c)-v_{f}(x, c)\right)=-\infty
$$


Отсюда следует существование и единственность решения $c_{0}$ уравнения (3).

Обозначим

$$
p(x, c)=\sup \left\{\frac{|c-f(y)|}{d(x, y)} \mid y \in D\right\} .
$$

Так как $p(x, c)=\max \left\{u_{f}(x, c), v_{f}(x, c)\right\}$, то при $c>c_{0}$

$$
p(x, c)=u_{f}(x, c)>u_{f}\left(x, c_{0}\right)=p\left(x, c_{0}\right),
$$

а при $c<c_{0}$

$$
p(x, c)=v_{f}(x, c)>v_{f}\left(x, c_{0}\right)=p\left(x, c_{0}\right) .
$$

Неравенство (4) доказано. Лемма доказана.

Лемма 2. Пусть $f, f_{1}$ - ограниченные на $D$ функиии, $a b \in \mathbb{R}$. Тогда

1) если $f \leqslant f_{1}$, mo $u_{f} \geqslant u_{f_{1}} u v_{f} \leqslant v_{f_{1}}$;

2) $u_{f+b}(x, c)=u_{f}(x, c-b), v_{f+b}(x, c)=v_{f}(x, c-b)$;

3) $n р u \quad a \geqslant 0 \quad u_{a f}(x, a c)=a \cdot u_{f}(x, c), v_{a f}(x, a c)=a \cdot v_{f}(x, c)$;

4) при $a<0 \quad u_{a f}(x, a c)=-a \cdot v_{f}(x, c), v_{a f}(x, a c)=-a \cdot u_{f}(x, c)$.

Все утверждения непосредственно следуют из определений $u_{f}$ и $v_{f}$.

3. Оператор продолжения. Определим функцию $g: X \rightarrow \mathbb{R}$, которая является продолжением функции $f$ на множество $X$ :

$$
g(x)= \begin{cases}f(x), & \text { если } x \in D, \\ \frac{1}{2}\left(\varlimsup_{\substack{y \rightarrow x \\ y \in D}} f(y)+\lim _{\substack{y \rightarrow x \\ y \in D}} f(y)\right), & \text { если } x \notin \bar{D} \backslash D, \\ c_{0}(x), & \text { если } x \in X \backslash \bar{D},\end{cases}
$$

где $c_{0}(x)$ - определено в лемме 1 . Равенство $E(f)=g$ определяет оператор продолжения $E$.

ПримеР. Пусть $X=[0,2], d(x, y)=|x-y|, D=\{0,1,2\}$ и на $D$ заданы две функции $f_{\text {и }} f_{1}: f(0)=0, f(1)=0, f(2)=1, f_{1}(0)=1, f_{1}(1)=0, f_{1}(2)=0$. Тогда

$$
E(f)=\left\{\begin{array}{ll}
\frac{x}{2}, & 0 \leqslant x<\frac{1}{2}, \\
\frac{1-x}{3-2 x}, & \frac{1}{2} \leqslant x<1, \\
x-1, & 1 \leqslant x \leqslant 2,
\end{array} \quad E\left(f_{1}\right)= \begin{cases}1-x, & 0 \leqslant x<1, \\
\frac{x-1}{2 x-1}, & 1 \leqslant x<\frac{3}{2}, \\
1-\frac{x}{2}, & \frac{3}{2} \leqslant x \leqslant 2 .\end{cases}\right.
$$

Отметим, что $E\left(f+f_{1}\right) \neq E(f)+E\left(f_{1}\right)$, т.е. оператор $E$ не является линейным.

Tеорема 1. Пусть $f, f_{1}$ - ограниченные на $D$ функиии, $a, b \in \mathbb{R}, g=E(f)$, $g_{1}=E\left(f_{1}\right)$. Oператор $E$ обладает следующими свойствами:

1) оператор сохраняет верхнюю и нижнюю грани значений функиии:

$$
\inf \{f(y) \mid y \in D\} \leqslant g(x) \leqslant \sup \{f(y) \mid y \in D\} \quad \forall x \in D
$$

2) относительная константа Липиица функиии $g$ в любой точке $x \in X \backslash \bar{D}$ не превосходит такой же константы для любого другого продолжения функиии $f$ на множество $X$;

3) $E(a f+b)=a E(f)+b$;

4) если $f(x) \leqslant f_{1}(x) \quad \forall x \in D$, mo $g(x) \leqslant g_{1}(x) \quad \forall x \in X$. 
ДоКАЗАТЕЛЬСТво. Свойства 1), 2) непосредственно следуют из леммы 1 , свойства 3), 4) - из леммы 2.

4. Вспомогательные свойства оператора продолжения. Для $x \in X \backslash \bar{D}$ определим величину

$$
L(x)=\sup \left\{\frac{|g(x)-f(z)|}{d(x, z)} \mid z \in D\right\},
$$

которая будет использоваться при исследовании свойств функции $g=E(f)$.

Лемма 3. Если $x \in X \backslash \bar{D}, m o$

$$
L(x)=\sup \left\{\frac{f(z)-f(y)}{d(x, y)+d(x, z)} \mid y, z \in D\right\}
$$

ДоКАЗАТЕЛЬСТВо. Из леммы 1 следует, что

$$
L(x)=\sup \left\{\frac{g(x)-f(y)}{d(x, y)} \mid y \in D\right\}=\sup \left\{\frac{f(z)-g(x)}{d(x, z)} \mid z \in D\right\} .
$$

Следовательно, $\forall y, z \in D$

$$
L(x) d(x, y) \geqslant g(x)-f(y), \quad L(x) d(x, z) \geqslant f(z)-g(x) .
$$

Отсюда

$$
L(x) \geqslant \frac{f(z)-f(y)}{d(x, y)+d(x, z)} .
$$

Из (6) следует, что $\forall \varepsilon>0 \exists y, z \in D$ такие, что

$$
\frac{g(x)-f(y)}{d(x, y)} \geqslant L(x)-\varepsilon, \quad \frac{f(z)-g(x)}{d(x, z)} \geqslant L(x)-\varepsilon .
$$

Получаем

$$
\frac{f(z)-f(y)}{d(x, y)+d(x, z)} \geqslant L(x)-\varepsilon .
$$

Так как $\varepsilon$ может быть выбрано произвольно малым, то

$$
\sup \left\{\frac{f(z)-f(y)}{d(x, y)+d(x, z)} \mid y, z \in D\right\} \geqslant L(x) \text {. }
$$

Лемма доказана.

Лемма 4. Пусть $x, y \in \bar{D}$, тогда

$$
\frac{|g(x)-g(y)|}{d(x, y)} \leqslant \min \{L(x), L(y)\} .
$$


ДоКАЗАТЕЛЬСтво. Пусть для определенности $L(x) \leqslant L(y)$ и $g(x) \geqslant g(y)$. Из определения $L(y)$ следует, что $\forall \varepsilon>0 \exists z \in D$ такое, что

$$
g(y)-f(z) \geqslant(L(y)-\varepsilon) d(y, z) .
$$

Из неравенства

$$
g(x)-f(z) \leqslant L(x) d(x, z)
$$

вычтем (7). Получаем

$$
\begin{aligned}
g(x)-g(y) & \leqslant L(x) d(x, z)-L(y) d(y, z)+\varepsilon d(y, z) \\
& \leqslant L(x)(d(x, z)-d(y, z))+\varepsilon d(y, z) \\
& \leqslant L(x) d(x, y)+\varepsilon \sup \{d(y, z) \mid z \in D\} .
\end{aligned}
$$

Так как $\varepsilon$ может быть выбрано произвольно малым, получаем

$$
\frac{|g(x)-g(y)|}{d(x, y)} \leqslant L(x) .
$$

Лемма доказана.

СлеДСтвиЕ 1. Если $x \in X \backslash \bar{D} u g=E(f), m o k(g, x)=L(x)$.

ДоКАЗАТЕЛЬСТво. Из определений $k(g, x)$ и $L(x)$ следует

$$
k(g, x)=\max \left\{L(x), k_{1}(x), k_{2}(x)\right\}
$$

где

$$
\begin{aligned}
& k_{1}(x)=\sup \left\{\frac{|g(x)-g(y)|}{d(x, y)} \mid y \in X \backslash \bar{D}\right\}, \\
& k_{2}(x)=\sup \left\{\frac{|g(x)-g(y)|}{d(x, y)} \mid y \in \bar{D} \backslash D\right\} .
\end{aligned}
$$

Из леммы 3 следует, что $k_{1}(x) \leqslant L(x)$. Поэтому достаточно показать, что $k_{2}(x) \leqslant L(x)$. Пусть $y \in \bar{D} \backslash D$ и для определенности $g(x) \geqslant g(y)$. Тогда

$$
\begin{aligned}
g(x)-g(y) & =g(x)-\frac{1}{2}\left(\varlimsup_{\substack{z \rightarrow y \\
z \in D}} f(z)+\varliminf_{\substack{z \rightarrow y \\
z \in D}} f(z)\right) \\
& \leqslant g(x)-\varliminf_{\substack{z \rightarrow y \\
z \in D}} f(z)=\varliminf_{\substack{z \rightarrow y \\
z \in D}}(g(x)-f(z)) \\
& \leqslant \varliminf_{\substack{z \rightarrow y \\
z \in D}} L(x) d(x, z)=L(x) d(x, y) .
\end{aligned}
$$

\section{5. Основной результат.}

Теорема 2. Пусть $\omega: \mathbb{R}_{+} \rightarrow \mathbb{R}_{+}-$непрерьвная, неубввающая функиия такая, что функиия $\omega(t) / t$ не возрастает. Eсли $f \in H(D, \omega)$, mо $g=E(f) \in H(X, \omega)$. 
ДоКАЗАТЕЛЬСТво. Покажем, что при $x \in X \backslash \bar{D}$ и $y \in D$

$$
|f(y)-g(x)| \leqslant \omega(d(x, y)) .
$$

Пусть для определенности $g(x) \leqslant f(y)$. Из определения $L(x)$ следует, что $\forall \varepsilon>0 \exists z \in D$ такое, что

$$
\frac{g(x)-f(z)}{d(x, z)} \geqslant L(x)-\varepsilon .
$$

Из неравенств $f(y)-g(x) \leqslant L(x) d(x, y)$ и $(9)$ получаем

$$
\begin{gathered}
f(y)-g(x) \leqslant\left(\frac{g(x)-f(z)}{d(x, z)}+\varepsilon\right) d(x, y), \\
(f(y)-g(x)) d(x, z) \leqslant(g(x)-f(z)) d(x, y)+\varepsilon d(x, y) d(x, z) .
\end{gathered}
$$

Прибавим к обеим частям последнего неравенства величину $(f(y)-g(x)) d(x, y)$. Получаем

$$
\begin{aligned}
(f(y)-g(x))(d(x, z) & +d(x, y)) \leqslant(f(y)-f(z)) d(x, y)+\varepsilon d(x, y) d(x, z), \\
f(y)-g(x) & \leqslant \frac{(f(y)-f(z)) d(x, y)}{d(x, z)+d(x, y)}+\frac{\varepsilon d(x, y) d(x, z)}{d(x, z)+d(x, y)} \\
& \leqslant \frac{\omega(d(y, z)) d(x, y)}{d(x, z)+d(x, y)}+\varepsilon d(x, y) .
\end{aligned}
$$

Так как функция $\omega$ не убывает, имеем

$$
\omega(d(y, z)) \leqslant \omega(d(x, y)+d(x, z)) .
$$

Так как функция $\omega(t) / t$ не возрастает, имеем

$$
\frac{\omega(d(x, y)+d(x, z))}{d(x, z)+d(x, y)} \leqslant \frac{\omega(d(x, y))}{d(x, y)} .
$$

Преобразуя (10) с учетом (11) и (12), получаем

$$
f(y)-g(x) \leqslant \omega(d(x, y))+\varepsilon d(x, y) .
$$

Так как $\varepsilon$ может быть выбрано произвольно малым, (8) доказано.

Покажем, что при $x, y \in X \backslash \bar{D}$

$$
|g(y)-g(x)| \leqslant \omega(d(x, y)) .
$$

Пусть для определенности $g(x) \leqslant g(y)$. Из определения величин $L(x), L(y)$ следует, что $\forall \varepsilon>0 \exists z, s \in D$ такие, что

$$
\begin{aligned}
& \frac{g(x)-f(z)}{d(x, z)} \geqslant L(x)-\varepsilon, \\
& \frac{f(s)-g(y)}{d(y, s)} \geqslant L(y)-\varepsilon .
\end{aligned}
$$


Воспользуемся неравенством

$$
\min \left(\frac{a_{1}}{b_{1}}, \frac{a_{2}}{b_{2}}\right) \leqslant \frac{a_{1}+a_{2}}{b_{1}+b_{2}},
$$

где $a_{1}, b_{1}, a_{2}, b_{2}$ - положительные числа. Из $(14),(15)$ получаем

$$
\min \{L(x), L(y)\} \leqslant \frac{f(s)-g(y)+g(x)-f(z)}{d(x, z)+d(y, s)}+\varepsilon .
$$

Из леммы 4 получаем

$$
\begin{gathered}
g(y)-g(x) \leqslant \min (L(x), L(y)) d(x, y) \\
\leqslant \frac{(f(s)-g(y)+g(x)-f(z)) d(x, y)}{d(x, z)+d(y, s)}+\varepsilon d(x, y), \\
(g(y)-g(x))(d(x, z)+d(y, s)) \leqslant(f(s)-g(y)+g(x)-f(z)) d(x, y) \\
+\varepsilon d(x, y)(d(x, z)+d(y, s)) .
\end{gathered}
$$

Прибавив к обеим частям последнего неравенства величину $(g(y)-g(x)) d(x, y)$, получаем

$$
\begin{aligned}
&(g(y)-g(x))(d(x, z)+d(x, y)+d(y, s)) \leqslant(f(s)-f(z)) d(x, y) \\
&+\varepsilon d(x, y)(d(x, z)+d(y, s)), \\
& g(y)-g(x) \leqslant \frac{(f(y)-f(z)) d(x, y)}{d(x, z)+d(x, y)+d(y, s)}+ \frac{\varepsilon d(x, y)(d(x, z)+d(y, s))}{d(x, z)+d(x, y)+d(y, s)} \\
& \leqslant \frac{\omega(d(s, z)) d(x, y)}{d(x, z)+d(x, y)+d(y, s)}+\varepsilon d(x, y) .
\end{aligned}
$$

Так как функция $\omega$ не убывает, имеем

$$
\omega(d(s, z)) \leqslant \omega(d(x, z)+d(x, y)+d(y, s)) .
$$

Так как функция $\omega(t) / t$ не возрастает, имеем

$$
\frac{\omega(d(x, z)+d(x, y)+d(y, s))}{d(x, z)+d(x, y)+d(y, s)} \leqslant \frac{\omega(d(x, y))}{d(x, y)} .
$$

Преобразуя (16) с учетом (17) и (18), получаем

$$
g(y)-g(x) \leqslant \omega(d(x, y))+\varepsilon d(x, y) .
$$

Так как $\varepsilon$ может быть выбрано произвольно малым, (13) доказано.

Докажем (13) в случае, когда $x \in X \backslash \bar{D}, y \in \bar{D} \backslash D$. Пусть для определенности $g(x) \leqslant g(y)$. Получаем

$$
\begin{aligned}
& g(y)-g(x)=\frac{1}{2}\left(\varlimsup_{\substack{z \rightarrow y \\
z \in D}} f(z)+\lim _{\substack{z \rightarrow y \\
z \in D}} f(z)\right)-g(x) \\
& \leqslant \varlimsup_{\substack{z \rightarrow y \\
z \in D}}(f(z)-g(x)) \leqslant \varlimsup_{\substack{z \rightarrow y \\
z \in D}} \omega(d(z, x))=\lim _{t \rightarrow d(y, x)} \omega(t)=\omega(d(x, y)) .
\end{aligned}
$$

Аналогично доказьвается (13) в случае, когда $x, y \in \bar{D} \backslash D$. Теорема доказана. 
СлЕДСтвиЕ 2. Если $m \geqslant 0, f \in m * \operatorname{Lip}(D), m o g \in m * \operatorname{Lip}(X)$.

Если функция $f$ равномерно непрерьвна на $D$, то в качестве функции $\omega$ может быть взята наименьшая вогнутая мажоранта функции

$$
\omega_{1}(f, t)=\sup \{|f(x)-f(y)| \mid x, y \in D, d(x, y) \leqslant t\}
$$

В этом случае $\omega$ удовлетворяет условиям теоремы 2 и является модулем непрерывности [4]. В этом смысле оператор продолжения $E$ сохраняет модуль непрерьвности.

Из теорем 1 и 2 следует утверждение более сильное, чем утверждение 3 работы [6], применяемое при исследовании пространства липшицевых функций на $(X, d)$.

СЛЕДСТВИЕ 3. Пусть $\omega(t)$ является модулем непрерывности и удовлетворяет условиям теоремы 2. Тогда для любого конечного $D \subset X$ и любой функиии $f: D \rightarrow \mathbb{R}$ функиия $g=E(f)$ такова, что

$$
\max _{x \in D}|f(x)| \leqslant \sup _{x \in X}|g(x)|, \quad g \in M_{1} * \operatorname{Lip}(X) \cap H\left(X, M_{2} \cdot \omega\right)
$$

$2 \partial e$

$$
\begin{aligned}
& M_{1}=\max \left\{\frac{f(x)-f(y)}{d(x, y)} \mid x, y \in D, x \neq y\right\}, \\
& M_{2}=\max \left\{\frac{f(x)-f(y)}{\omega(d(x, y))} \mid x, y \in D, x \neq y\right\} .
\end{aligned}
$$

Сравнивая операторы продолжения $E_{1}, E_{2}$ (см. (1), (2)) с оператором $E$, отметим, что $E_{1}, E_{2}$, вообще говоря, не обладают свойствами 1$\left.)-3\right)$ теоремы 1 . В отличие от $E_{1}, E_{2}$ оператор $E$ определяется только через функцию $f$, без привлечения функции $\omega$. Оператор $E$ корректно определен даже в том случае, если $f$ ограничена, но разрьвна на $D$. Представление о поведении функции $g=E(f)$ вне множества $\bar{D}$ дает

Теорема 3. Пусть $\varepsilon>0, D_{\varepsilon}-\varepsilon$-окрестность множества $D$,

$$
m=\frac{1}{2 \varepsilon}-\sup \{|f(x)-f(y)| \mid x, y \in D\} .
$$

Tогда $g \mid\left(X \backslash D_{\varepsilon}\right) \in m * \operatorname{Lip}\left(X \backslash D_{\varepsilon}\right)$.

Доказательство следует из леммы 3 и следствия 1.

6. О следе локально липшицевой функции. В качествеприложения оператора продолжения $E$ рассмотрим вопрос о следе локально липшицевой функции на компактном подмножестве, поднятьй в [2].

Далее $(X, d)$ - линейно связное метрическое пространство, причем метрика $d$ является внутренней. Последнее означает, что расстояние между любыми двумя точками есть точная нижняя грань длин кривых, соединяющих эти точки. Будем использовать обозначение $B(x, r)=\{y \in X \mid d(x, y)<r\}$. Если $S$ - дуга конечной длины в $X$, то через $|S|$ будем обозначать ее длину. 
Tеорема 4. Пусть $D$ - компактное подмножество $X$ без внутренних точек $u X \backslash D$ линейно связно. Для того чтобы непрерывная функиия $f: D \rightarrow \mathbb{R}$ была следом некоторой функиии $h: X \rightarrow \mathbb{R}$, удовлетворяющей условию

$$
\varlimsup_{y \rightarrow x} \frac{|h(x)-h(y)|}{d(x, y)}<1 \quad \forall x \in X \backslash D,
$$

необходимо и достаточно, чтобъ $\forall y, z \in D, \forall x \in X \backslash D$

$$
|f(y)-f(z)|<d(x, y)+d(x, z) .
$$

При доказательстве потребуется следующее утверждение.

Лемма 5. Пусть точки $z_{1}, z_{2} \in X$ соединены дугой $S$ конечной длины и в каждой точке $x$ әтой дуги функиия $h: X \rightarrow \mathbb{R}$ удовлетворяет условию

$$
\varlimsup_{y \rightarrow x} \frac{|h(x)-h(y)|}{d(x, y)} \leqslant m .
$$

Тогда $\left|h\left(z_{1}\right)-h\left(z_{2}\right)\right| \leqslant m \cdot|S|$.

ДокаЗАтЕльство. Выберем $\varepsilon>0$. Каждой точке $x$ на дуге $S$ поставим в соответствие число $r(x)>0$ такое, что если $d(x, y) \leqslant 2 r(x)$, то $|h(x)-h(y)| \leqslant d(x, y)(m+\varepsilon)$. Множество $\{B(x, r(x)) \mid x \in S\}$ образует открытое покрытие $S$. В силу компактности дуги в покрытии существует конечное подпокрытие. Зафиксируем подпокрытие и перенумеруем в нем центры шаров в порядке следования по дуге от $z_{1} \mathrm{\kappa} z_{2}$. В полученной конечной последовательности шаров произведем отбор таким образом, чтобы каждая пара соседних шаров имела непустое пересечение и при этом точка $z_{1}$ лежала в первом шаре, а точка $z_{2}$ - в последнем. Такой отбор возможен в силу того, что шары образуют покрытие дуги. Центры отобранных шаров обозначим через $x_{i}, i=1,2, \ldots, n$. Получаем

$$
\begin{aligned}
\left|h\left(z_{1}\right)-h\left(z_{2}\right)\right| & =\left|h\left(z_{1}\right)-h\left(x_{1}\right)+h\left(x_{1}\right)-h\left(x_{2}\right)+\cdots+h\left(x_{n}\right)-h\left(z_{2}\right)\right| \\
& \leqslant(m+\varepsilon)\left(d\left(z_{1}, x_{1}\right)+d\left(x_{1}, x_{2}\right)+\cdots+d\left(x_{n-1}, x_{n}\right)+d\left(x_{n}, z_{2}\right)\right) \\
& \leqslant(m+\varepsilon)|S| .
\end{aligned}
$$

Так как $\varepsilon$ может быть выбрано произвольно малым, лемма доказана.

ДокаЗАТЕЛЬСтво теоремЫ 4. Достаточность. Возьмем $h=E(f)$. Тогда в силу следствия 1 и леммы 3 при $x \in X \backslash D$

$$
\varlimsup_{y \rightarrow x} \frac{|g(x)-g(y)|}{d(x, y)} \leqslant L(x)=\sup \left\{\frac{f(z)-f(y)}{d(x, y)+d(x, z)} \mid y, z \in D\right\} .
$$

Так как $D$ компактно, $f$ непрерывна и $\inf \{d(x, y) \mid y \in D\}>0$, то верхняя грань в последнем выражении достигается на некоторых $y, z \in D$. В силу (19) $L(x)<1$.

Необходимость. Пусть $x \in X \backslash D$. Выберем $\varepsilon>0$ так, чтобы $B(x, \varepsilon)$ лежал в $X \backslash D$ и $\forall x_{1} \in B(x, \varepsilon)$ вьполнялось

$$
\frac{\left|h(x)-h\left(x_{1}\right)\right|}{d\left(x, x_{1}\right)} \leqslant a<1,
$$


где

$$
a=\frac{1}{2}+\frac{1}{2} \varlimsup_{y \rightarrow x} \frac{|h(x)-h(y)|}{d(x, y)} .
$$

Соединим $x$ с точками $y, z \in D$ дугами $S_{y}, S_{z} \subset X \backslash D$ соответственно. Через $p$ обозначим одну из точек пересечения дуги $S_{y}$ со сферой $\{t \mid t \in X, d(t, x)=\varepsilon / 2\}$. Через $q$ обозначим одну из точек пересечения $S_{z}$ с этой же сферой. Точка $p$ разбивает дугу $S_{y}$ на две части $-S_{y p}$ и $S_{p x}$. Тогда $q$ разбивает дугу $S_{z}$ на две части $-S_{z q}$ и $S_{q x}$. Все указанные дуги и точки существуют в силу линейной связности $X \backslash D$. Используя лемму 5 , получаем

$$
\begin{aligned}
f(y)-f(z) & \leqslant(f(y)-h(p))+(h(p)-h(x))+(h(x)-h(q))+(h(q)-f(z)) \\
& \leqslant\left|S_{y p}\right|+2 a \cdot \frac{\varepsilon}{2}+\left|S_{z q}\right|=\left|S_{y}\right|-\left|S_{p x}\right|+\left|S_{z}\right|-\left|S_{q x}\right|+a \cdot \varepsilon \\
& \leqslant\left|S_{y}\right|+\left|S_{z}\right|-(1-a) \varepsilon .
\end{aligned}
$$

Так как дуги могут быть выбраны таким образом, чтобы их длины были сколь угодно близки к расстояниям между их концами, из (20) получаем

$$
f(y)-f(z) \leqslant d(y, x)+d(z, x)-(1-a) \varepsilon<d(y, x)+d(z, x) .
$$

В отличие от [2] в теоремене требуется локальная компактность пространства $(X, d)$.

Институт технической кибернетики

Поступило

АН Беларуси

20.10 .94

\section{СПИСОК ЦИТИРОВАННОЙ ЛИТЕРАТУРЫ}

[1] Федерер Г. Геометрическая теория меры. М.: Наука, 1987.

[2] Клячин А. А., Миклюков В. М. Следы функций с пространственноподобными графиками и задача о продолжении функций при ограничениях на градиент // Матем. сб. 1992. Т. 183. №7. C. 49-64.

[3] Mustata C. Extension of Holder functions and some related problems of best approximation // Faculty of Mathematics. Research Seminars. Preprint № 7: Babes-Bolyai University, 1991. P. 71-86.

[4] Тиман А.Ф. Теория приближения функций действительного переменного. М.: Физматгиз, 1960.

[5] Czipcer J., Cheher L. Extention of functions satisfying a Lipschitz condition // Acta Math. Acad. Sci. Hung. 1955. V. 6. №1-2. P. 213-220.

[6] Hanin L. G. Kantorovich-Rubinshtein norm and its application in the theory of Lipschitz spaces // Proc. Amer. Math. Soc. 1992. V. 115. № 2. P. 345-352. 